of operation, or believe that the patient can be cured by medical means.

"To prevent disappointment it is best to look on tuberculin as of assistance in the treatment of tuberculosis of the kidney and not as a specific remedy therefor." In forming an opinion of its value we must use as a base the reports of those who have had the patience to use it, over long periods of time, in numerous cases that have answered to the requirements of a perfect diagnosis: the direct demonstration of tubercle bacilli in an acid purulent urine received in a sterile container from a sterile ureteral catheter introduced into the pelvis of the kidney of a person afflicted with polyuria, dysuria or both; or the development in a guinea-pig inoctulated with the sediment of this urine, of tuberculosis at the point of inoculation.

The evidence of experienced operators, who have frequently nephrectomized such patients with a low mortality both immediate and remote, yet who have had the courage thoroughly to test out the merits of tuberculin, is the best evidence of all, for they know and appreciate better than others the unreliable history of the disease and its unaccountable vagaries-how the individual develops a marked resistance to reinfection both from within and without, accounting for the slow progress of renal tuberculosis, its remissions of disturbing symptoms, and sometimes of apparent cure for months and for years, by the encapsulation and sealing up of the abscess cavity, thus introducing an element of doubt always into the origin of any favorable results following the use of tuberculin. Its use as a remedy in tuberculosis of the kidney can never be other than empirical. Many who have sung the praises of its curative powers have not always been very careful to make sure that their cases were tuberculosis of the kidiney and were satisfied to report a cure when their patients increased in weight and the vesical symptoms decreased in intensity. There is no case on record that I have been able to find, that answers the full requirements of a cure of tuberculosis of the kidney by tuberculin; and yet there are many intelligent urologic surgeons who believe that it is possible if the disease is recognized early that it may be cured by hygienic and climatic measures if aided by tuberculin, and all agree that it is at least a useful placebo in bilateral renal tuberculosis, and in those cases in which one kidney having been removed the remaining one is attacked by the bacillus. Firmly convinced that in unilateral nodular caseous tuberculosis of the kidney, in the presence of a sound companion and without the existence of any other contraindication thereto, nephrectomy is the only remedy that promises a permanent cure, I have found it very diffcult to convince the sick man and his friends of the benignancy of the operation in skilled hands and the necessity therefor, and so have been compelled to treat very many cases against my judgment as what was best, by tuberculin, duotal and hygienic measures. The clinical results in a few instances have been astonishing and in many fairly satisfactory, and were it not for the inexorable average history of unilateral tuberculosis of the kidney, did we not know that the walled-in abscess may contain living bacilli that hibernate for years only to be released by some mechanical insult or some lowered resistance giving rise to metastatic autoinfection again, I might well believe I had seen cures effected by tuberculin.

It is not a matter of much importance what partic. ular preparation of tuberculin is used. The Belgians, and many of the French, use Deny's B. F. In Germany and America Koch's O. T. and N. T. and B. E. are in most general use.

The dose, however, is not a matter of indifference. It should be be small, always very small, at first. One that will equal from $1: 1,000,000$ to $1: 100,000 \mathrm{mg}$. of $O$. T. is always sufficient to start with and it should be raised very gradually, avoiding the occasioning of a local or general reaction. The intervals of its administration should be four or five days. Used in this way it helps many and injures none.

Lissner Building.

\section{ACUTE UNILATERAL HEMATOGENOUS INFECTIONS OF THE KIDNEY*}

JOHN H. CUNNINGHAM, JR., M.D. BOSTON

At the meeting of the American Association of Genito-Urinary Surgeons in June, 1912, I reported a series of cases of acute unilateral hematogenous infection of the kidney, some treated by nephrectomy, others by decapsulation or drainage, and another group by non-surgical measures.

At this time it is my desire to relate the subsequent course of these patients and to refer to others which have come under my observation since that time. Tuberculosis, the most common of all hemic infection of the kidney, is not to be considered.

Suppuration within the kidney parenchyma, as is well known, results from infection by one or. another of certain varieties of micro-organisms taking place usually in one or two ways, either as an ascending infective process from the lower urinary tract or brought to the kidney by the blood stream; yet rarely the kidney becomes acutely infected by adjacent septic foci or by penetrating wounds.

Ascending kidney infection is not the subject under consideration, and will not be considered, yet it must be stated that it is quite impossible in some instances of Bacillus coli infection of the kidney, to be certain that its origin is hemic and not ascending.

The acute renal infections of hematogenous origin are of two distinct classes: one induced by infective or toxemic conditions, for example, nearly all of the infections which give rise to general diseases such as diphtheria, scarlet fever, measles, small-pox, pneumonia, typhoid fever, etc. The disturbance within the kidney is due, in this class, to the action of toxic substances elaborated by the virus of the special disease which is in part eliminated through the kidneys. While the toxic substances of these acute general diseases conveyed to the kidney by the blood stream induce cellular necrosis, it is rare that suppuration occurs, and this type of hematogenous kidney disturbance remains medical rather than surgical.

It is the second, rather smaller, yet more serious, class of kidney infection of hematogenous origin, whereby the kidney is invaded by the micro-organisms themselves, resulting in acute inflammation and suppuration, that commands our attention from a surgical standpoint.

Acute suppurative nephritis of hematogenous origin is a condition resulting from the invasion of the kidney by certain micro-organisms, in particular by the

* Read before the Section on Genito-Lrinary Diseases at the SixtyFifth Annual Session of the American Medical Association, Atlanit City, N. J., June, 1914 
pyogenic cocci and the colon bacillus. It is theoretically of a metastatic nature, and when encountered in both kidneys is usually consecutive to septic endocarditis or other septic affections elsewhere in the body. When it occurs in but one of the two kidneys the primary focus from which the organisms find their way to the kidney has seldom been determined; yet there are some instances in which acute tonsillitis, osteomyelitis and similar acute diseases of pyogenic origin may be correlated with an acute hemic infection of the kidney. Israel feels that he has been able to trace such renal infections to such simple infections as furuncles and paronychia.

\section{PATHOLOGY}

The pathology of acute unilateral hematogenous infections of the kidney is quite different from the acute bilateral kidney infections. The latter are an expression of a general septicemia or pyemia in which the kidneys participate. The former, the acute unilateral kidney infections of hematogenous origin, are not a part of general pyemia, but find their origin in the lodging of a minute embolus carrying one or several organisms in the terminal vessels of the kidney. It is believed that the process may be started by a single micro-organism.

The condition starts not by multiple simultaneous points of infections throughout the kidney, as the lesions observed in the kidneys removed at operation might suggest, but as a single focus in the cortex of the organ. When the organisms settle in the minute capillary vessels of the glomerulus, an inflammatory reaction is set up around the glomerulus, and the surrounding tissue is infiltrated with the products of inflammation. From the primary focus of the infection other areas are secondarily involved by the infectious material gaining access to other tubules, to the lymph spaces and through the vessels in the connective tissue stroma. The infection thus is more or less rapidly spread through these channels to other parts of the organ.

The pathologic process originating as described above is of two distinct types: (1) abscess formation, and (2) a diffuse inflammatory process without breaking doron of tissue.

In the former type of the disease, abscess formation, the kidney is found somewhat enlarged from congestion, and disseminated through its substance are small separate foci of suppuration or miliary abscesses surrounded by areas of congestion. In the early stages of the disease these minute abscesses are often observed in the cortex just beneath the capsule. From here other parts of the organ are secondarily involved, as just mentioned. If the process continues, these foci may enlarge, coalesce and form abscesses of larger size which may rupture the kidney capsule, and invade the perirenal fat, resulting in a perinephritic abscess. For the infection to complete this cycle, the resistance of the individual must be great and the virulence of the infecting organisms comparatively slight, as the systemic toxemia from this disease is rapid and death ustually occurs before the process develops to this stage. The organisms producing this form of kidney inflammation are the pyogenic cocci, the Staphylococcus pyogones albus and aureus, and the Streptococcus pyogenes.

The second type of the disease, a diffuse inflammatory process spreading through the kidney substances, but not resulting in focal abscesses or solution of tissue, has the same sequence of events in its pathology when of hematogenous origin. This type of the disease presents a pathologic condition more commonly the result of an ascending infection, but is encountered as a process of hematogenous origin and results not from an infection of the organ by the pyogenic cocci, as does the abscess type, but by the colon bacillus.

The difference in the severity of the process depends on the lesser virulence of the infecting organisms. The organ is found to be enlarged from congestion. There may be subcapsular areas of more or less acute reddening, and on section the organ is found presenting irregular, circumscribed, acutely reddened or pale yellowish areas involving the cortex and pyramids or involving nearly the whole organ, but without solution of tissue. In the early stage of the process these isolated areas are distinct and are seen as irregular, acutely reddened patches with the edges streaked downward into the pyramids. As the inflammatory process subsides, these areas lose the acute red color and are replaced by tissue of a paler character. In some instances the whole kidney may present an acutely reddened surface, owing to the acute process involving the whole organ. This form of the disease undergoes resolution without focal degeneration of tissues, and when seen after the acute process has subsided, presents paler areas, dependent on the stage of the connective tissue repair process.

By studying the clinical course of these two forms of the disease, it becomes evident that the clinical signs differ in some respects, and each has a definite relation with these two different pathologic conditions. The subjective and objective signs which the patient presents should suggest the type of process at work in the affected kidney, and these two types of infection should be recognized on inspection of the diseased organ at the time of operation, for experience has shown that these different types of infection do not require the same form of treatment. While the kidney containing numerous focal abscesses requires nephrectomy, less radical measures suffice in the diffuse, non-suppurative form of infection.

From the clinical aspect of the subject the picture which unilateral multiple abscess formation produces is an acute disease which may or may not be ushered in by chill. There is a rapid rise of temperature to from 102 to $105 \mathrm{~F}$, and an early acceleration of pulse rate usually to 120 or higher, occurring often within twenty-four hours following the onset. Sepsis or toxemia and a high leukocytosis develop rapidly. Pain on the side of the affected organ, abdominal tenderness, muscular rigidity and spasm are found on the affected side. Lumbar tenderness, rigidity and spasm are pathognomonic signs of the disease. The abdominal signs are similar to those occurring in acute affection of the appendix and gall-bladder, for which this disease of the kidney is often, if not usually, mistaken. Unfortunately, the urine in this disease does not excite suspicion of the true condition, as it is usually quite normal in its gross and microscopic appearance, and genearlly contains but little albumin and only a small amount of pus and blood-cells, because the abscesses find no avenue of escape through the collecting tubules, which are either plugged by the product of inflammation or compressed by the infiltrated tissue surrounding them.

When the infection is severe, the patient grows progressively and rapidly worse, the toxemia becomes profound, delirium sets in and there is a fatal termination within a few days unless the body is rid of the 
infected organ. Of this form of the disease I have observed five cases. The following cases illustrate this type of the disease:

CASE 1.-Woman, aged 38, entered the gynecologic service of the Boston City Hospital under the care of Dr. Franklin Newell and Dr. Ernest Young, March 27, 1912. The past history was negative, with the exception that one year ago both tubes, ovaries and appendix were removed for acute inflammation. The present illness was characterized by severe pains in the right kidney region, chills, fever and vomiting beginning three days before entrance and gradually increasing in severity. The temperature at entrance was 103.4 and the pulse 120 , leukocytosis 28,000 . The general condition of the patient gave every evidence of good health prior to the onset of the present illness. The pliysical examiration showed a flushed face, throat and lungs negative, anemic murmur in the heart. The abdomen was not distended, but showed marked tenderness in the right side, less so on the left. Exquisite tenderness in the right upper quadrant and in the right loin. There was rigidity and spasm of the right rectus and lumbar spasms. Vaginal examination was negative. The urine was turbid, 1.020 , acid, slight trace of albumin, no sugar or bile. The sediment was small in amount and consisted of pus, an occasional blood corpuscle and epithelial cell. The patient's condition became progressively worse during the following two days, and on the third day after entrance (six days after onset of the trouble) was extremely bad. At this time I saw the patient in consultation with Dr. Young. Her appearance was that of profound sepsis, pulse 130 of poor quality, temperature 103. There was a rigidity of the right side of the abdomen, with marked tenderness over the right kidney in front and in back. The urine at this time was only turbid and contained little pus. The leukocytosis was 18,000 . An operation was advised as the only chance for life, although the risk was considered great.

A lumbar incision was made, the perinephritic fat found infiltrated with cloudy serum but no pus. The kidney was exposed and delivered. It was twice normal size, congested, and over the surface were scattered many focal abscesses from 1 to $5 \mathrm{~mm}$. in diameter. The pedicle was clamped and the kidney removed. No attempt was made to tie the pedicle on account of the poor condition of the patient, stimulation and salt solution being required during the operation. The clamp was left on the pedicle; the wound drained by a spiral tube with gauze and the wound partially closed.

Twenty-four hours after operation the patient's temperature dropped to 100 and the pulse to 110 . The improvement in appearance and general condition was perceptible. The clamp was removed from the kidney pedicle on the sixth day. The patient made an uneventful recovery.

The pathologic report was acute inflammation of the kidney, with multiple miliary abscesses from which Staphylococcus pyogenes was recovered.

Two years after operation this patient is living and well.

CASE 2.-Woman, aged 32, seen in consultation with Dr. Albert Griffiths and Dr. Nathaniel Thayer at the Swedish Hospital, Brooklyn, Sept. 2\%, 1907. Previous health had been excellent. For two days the patient had complained of pain in right kidney region. There had been a chill, rise of temperature to 103 , and urine was cloudy, containing 0.25 per cent. albumin and considerable sediment, consisting of pus, normal and abnormal blood, but no casts. The pain became more severe, and her general condition failed rapidly. When seen by me the general condition was poor, temperature 103.5, pulse 108, and urine as mentioned above. There was tenderness over the whole right side of the abdomen, rigidity and spasm of the right rectus. There was exquisite lumbar tenderness with spasm. The kidney was approached through a lumbar incision, the perirenal fat found to be edcmatous and adherent to the kidney capsule in the central third of the organ. The kidney when freed and delivered was found to be about one-third larger than normal and much congested. In the middle quarter an area about $11 / 2$ inches in length, of irregular surface, slightly harder than normal was found. This area extended over the anterior and posterior surfaces of the kidney to within about $3 / 4$ inch of the renal pelvis. The kidney was split in the convex border and the area observed on the surface was found to involve the central quarter of the kidney down to the renal pelvis. This area, darker than the surrounding tissue, contained several small foci of purulent material from 1 to 4 $\mathrm{mm}$. in diameter. The remainder of the kidney showed only congestion. A wedged-shaped piece including the central third of the kidney and passing about $1 / 2$ inch outside the infected area was resected. The upper and lower remaining thirds of the kidney were then brought together by mattress sutures. The wound was united around a tube and gage drain. The temperature dropped to normal on the second day following operation, and the abnormal elements disappeared from the urine. The patient left the hospital fifteen days after the operation with a small, clean, granulating wound. The improvement in general condition was rapid.

The pathologic report showed acute inflammation with multiple abscess formation. Staphylococi were demonstrated in the section, but the organism was not isolated. Dr. Griffiths reports that the patient has continued in good health since the operation (Sept. 27, 1907), now six years and nine months.

CASE 3.-Woman, aged 34, presented the following clinical history: About five months ago she had an illness characterized by fever, pain in right side of abdomen and back. It was considered appendicitis by the attending physician. Remained in bed two weeks, at which time the symptoms had disappeared. An indefinite pain remained in right kidney region, more noticeable after physical exertion. Three months ago had a similar acute attack.

Aug. 1, 1906, the patient was again taken acutely ill, and when seen by me had a temperature of 101.8 , pulse 110 , and a leukocytosis of 18,000 . The urine was negative except for a slight trace of albumin and a few pus and blood cells. There was severe pain in the right hypochondrium and acute tenderness and rigidity over the right rectus and in the right costovertebral angle. The appendix region was negative. During the following two days the temperature rose to 104 . pulse to 120 and white count to 22,000 . The urine remained the same. The pain, tenderness and rigidity became more marked and the patient began to look toxic. The diagnosis of acute inflammation of the kidney was made. The kidney was approached through a lumbar incision, the perirenal fat found edematous and the kidney about one-third larger than normal. Beneath the capsule were many small miliary abscesses. The pedicle was clamped and the kidney removed. The pedicle was tied and the wound closed with drainage. Within two days the temperature and pulse had fallen to normal and the general condition of the patient was much improved. The pathologic report was acute inflammation of the kidney with abscess formation with areas of scar tissue.

This case is of special interest because of the presence in the kidney of areas of scar tissue which may be correlated with the previous attack of illness with renal symptoms, as related in the history, and suggests that resolution of an infection of this type may take place.

CASE 4.-Woman, aged 38, had had excellent health. Four days ago she was taken suddenly with severe pain in the right upper quadrant of the abdomen, accompanied by vomiting. She felt feverish and had several chills. When seen by me, Nov. 15 , 1908, the temperature was 102.4 , pulse 108 , white count 17,200 . In the costovertebral angle exquisite tenderness and marked muscular rigidity. In the right hypochondrium there was marked tenderness and rigidity, suggesting gall-bladder disease. Urine of high color, no bile, 1.014, acid, with a trace of albumin. The sediment was small in amount and contained many leukocytes, rare normal and abnormal blood-cells, hyaline and brown casts, occasionally fatty renal cells, and a few squamous epithelial cells. The patient remained in this condition without much change for four days, when it became evident that the toxemia was becoming pronounced, and the signs in the costovertebral 
region accentuated. The kidney was approached through the lumbar region, the perirenal fat found edematous, the kidney somewhat enlarged, and beneath the capsule could be seen many discrete abscesses about 2 to $4 \mathrm{~mm}$. in diameter. A nephrectomy was performed, the pedicle tied, the wound drained; the temperature and pulse fell to normal on the third day; the abnormal elements disappeared from the urine except for the presence of epithelial cells. The pathologic report was acute inflammation of the kidney with abscess formation.

The patient was last seen in 1910 (four years after operation). At that time she was well, had gained in weight and considered her general condition better than it had been for years.

CASE 5.-Boy, aged 17. Past history negative except severe tonsillitis seven days ago. Abdominal symptoms suggesting the peritonitis sometimes associated with tonsillitis appeared three days before patient was seen by me (Sept. 10, 1911). At that time the patient was acutely ill; temperature 103, pulse 110 . White count 22,000 . The tonsils were still acutely inflamed. There was tenderness over the whole abdomen and spasm on the right side. There was tenderness in the costovertebral space on the right. Urine 1.024, high, a large trace of albumin; a small amount of sediment, consisting of blood, large and small round cells and a few pus cells and granular casts. While the patient had been severely ill with tonsillitis from the onset, the attending physician believed the patient to be much more toxic since the onset of the abdominal symptoms. The patient was in a critical condition and it was believed that the right kidney had become acutely infected.

The right kidney was exposed througl the loin. The perirenal fat was edematous. The kidney was enlarged about one-third over normal, and scattered over its surface were numerous foci of suppuration from 2 to $5 \mathrm{~mm}$. in diameter. The kidney pedicle was clamped and tied, and the kidney removed. The wound was closed with drainage.

The pathologic diagnosis was acute inflammation of the kidney with abscess formation. The Staphylococcus albus and the Streptococcus pyogenes were found.

There was considerable improvement in the patient's condition during the following twenty-four hours, but he remained critically ill for ten days, when the temperature then reached normal. At this time the tonsillitis had subsided and the patient made a slow but uneventful recovery. Two years and six months after operation the patient was in excellent condition.

The clinical picture produced by a diffuse inflammatory process within the kidney without abscess formation differs from that with abscess formation chiefly in the severity of the symptoms and is the type of the disease most usually due to Bacillus coli infection and most difficult to distinguish from an ascending infection. The onset may be sudden, as in the former type, with acceleration of the pulse rate; and a leukocytosis is always present, often high. A chill may accompany the onset. Pain in the loin on the affected side is an early symptom, sometimes indefinite but often severe, varying according to the severity of the process. Tenderness over the kidney in the costovertebral angle, with some muscular rigidity and abdominal tenderness and rigidity on the affected side, is a constant sign. The chief feature differentiating this form of the disease from that of abscess formation is the lesser degree of progressive toxemia and the presence of more abnormal elements in the urine. These patients, while acutely ill, do not have the very severe and rapidly progressive toxic course which characterizes the other form of the disease. The illness after the first few days following the onset remains more or less stationary and may continue not unlike a beginning typhoid fever. The pain, tenderness and muscle rigidity may become gradually more pronounced, and the temperature, pulse and leukocytosis elevated, or the different signs may gradially decline. Remissions in the acute symptoms occur, but indefinite pain on the affected side usually persists indefinitely, and recurrences of the acute symptoms are not uncommon. The following cases are illustrative of this type of the disease:

CASE 6.-Woman, aged 24, gave a history of syphilis four years ago. Treated with mercury continuously for three years. Jan. 7, 1910, laparotomy was performed by another surgeon because of severe pain on the right side of the abdomen, associated with spasm and tenderness over the right rectus muscle. At this time the temperature was 102.5 . A few small cysts of the right ovary and a normal appendix were removed. A thorough exploration of the remaining abdominal organs proved negative. Following operation the pain in the right side persisted, the temperature ranged between 101 and 104, and the pulse around 120. The white count was 14,000. This condition persisted for about one week, when the temperature returned to normal, the pulse to 100 , and the tenderness became much less.

One week later the pain again became severe, the temperature rose to 102 , and the right side of the abdomen and back became very tender. Examination showed marked tenderness and involuntary spasm over the right side of the abdomen and back. White count was 23,000. The patient remained in this condition for about one week, when symptoms gradually subsided. The urine at this time showed but a trace of albumin, a small amount of pus, single and in clumps. A few small round cells and granular casts.

Two weeks later another acute attack of pain on the right side. Temperature rose to 102 , pulse to 120 . The twentyfour hour amount of urine at this time was 550 c.c. The sediment showed more pus, many small, round epithelial cells and a few hyaline and granular casts. Roentgen-ray examination was negative.

I first saw the patient in this attack. At this time there was general abdominal tenderness and spasm more marked over the right side. There was exquisite tenderness and spasm in the right costovertebral space. The general condition was only fair. The patient looked septic. The diagnosis of acute inflammation of the kidney was entertained.

An anterior incision was made, the peritoneum opened, the abdomen explored and found to be negative. The anterior incision was closed. The kidney was then approached through the loin. The perirenal fat was found adherent to the kidney and a hard nodule about the size of a Concord grape situated in the perirenal fat was removed. The kidney was slightly larger than normal and showed many red areas beneath the capsule. On section of the organ, similar areas were found scattered through the kidney substance. Nephrectomy was performed, the pedicle tied and the wound drained.

Four days following the nephrectomy the patient developed a right lobar pneumonia which a few days later involved the left side, and death occurred eleven days after operation. The wound at this time had healed except for a small granulating area.

The pathologic report showed acute inflammation of the kidney, with several areas showing connective tissue repair. The Bacillus coli was recovered from the tissues.

CASE 7.-Man, aged 31 , seen in consultation with Dr. Richard Collins at the Waltham Hospital, Jan. 25, 1910, since childhood has had a chronic discharge from the right ear. About ten years ago he had a severe attack of pain in the right kidney region, the facts regarding which are indefinite. Five years ago he had a similar attack and passed a small amount of blood in urine. He was confined to the bed for a short time. Four years ago he had a similar attack associated with fever, and frequent urination, and passed small amounts of blood in the urine several times.

Five days ago, after a hearty dinner, patient had a severe attack of pain in right side, associated with a chill and vomiting. $\mathrm{He}$ was then admitted to the Waltham Hospital, acutely ill, with pain on right side, temperature 102, pulse 104 . 
Acute symptoms gradually subsided, but pain in the back persisted. Roentgen-ray examination was negative. He was seen by me, Feb. 25, 1910. The patient's condition was as noted above. A cystoscopy performed at this time showed the bladder urine to be slightly cloudy and containing shreds. This urine was 1.020 in specific gravity, acid, and the sediment showed considerable pus, small round and many squamous epithelial cells. There was considerable fresh blood. Trigon was normal. Both ureteral orifices were normal in size and character. About $3 / 4$ inch above the right ureteral orifice was seen a group of eight or ten acutely injected areas from which bleeding had taken place and on which were adherent blood-clots. These areas were a little larger than a pin, and resembled early tuberculosis of the bladder. The remainder of the bladder was normal. Both ureters were catheterized. The urine collected from the two was equal in amount. The examination of the urine from the left side showed little abnormal; from the right side considerable pus and normal blood-cells; a few caudate and small round cells. Guinea-pigs inoculated for tuberculosis did not develop the disease.

Jan. 29, 1910, an exploratory examination of the kidney was made on account of the persistent pain and cystoscopic findings. The kidney was exposed through the loin. The perirenal fat was but slightly adherent to the kidney. Kidney was delivered and found slightly larger than normal. Beneath the capsules were seen a few grayish-white, contracted areas and a few acutely red areas. There was nothing abnormal in the remainder of the kidney or kidney pelvis. The kidney was split in Brödel's line. There were two acutely reddened areas in the upper half of the kidney. Believing the persistent pain to be due to these contracted areas, the kidney was removed, the pedicle tied and the wound drained.

The temperature, which was 101.5 at the time of operation, dropped to normal within twenty-four hours. The wound healed by first intention.

The pathologic report was an acute, diffuse inflammation of the kidney, with healed areas. The colon bacillus and Staphylococcus pyogenes aureus were recovered from the tissues. When last heard of, about one year following operation, the patient was reported to be well.

CaSE 8.-Woman, aged 43, seen in consultation with Dr. H. W. Barker and Dr. A. W. Tucker of Lynn; thirteen years ago had a severe illness, characterized by pain in the right side of abdomen and back, fever, chills and cloudy urine. She was in bed for several weeks. Since that time she had several similar attacks which confined her to bed for from two weeks to three months. The different physicians who have cared for her have considered that in these attacks she was suffering from "peritonitis." Some advised operation, which the patient refused. Between these attacks there has been a constant pain, varying in severity, in the left kidney region. Several Roentgen examinations have shown nothing abnormal. Four years ago the patient had a small amount of blood in the urine during one of these attacks. This occurred again two years ago. It never lasted more than a few days.

Feb. 2, 1912, the patient was cystoscoped by Dr. H. W. Baker and a collargol injection and roentgenogram were made of the left kidney. The cystoscopy showed the bladder to be normal in all respects, both ureteral orifices were normal, and the catheters were passed to each kidney. The specimen of urine collected showed that from the right side to be normal, and that from the left to contain a few leutocytes, normal red cells, and caudate epithêlial cells. The capacity of the renal pelvis was 14 c.c. The roentgenogram of the injected kidney pelvis showed it to be normal. No shadow appeared in the control plate.

Feb. 14, 1912, I again cystoscoped the patient, and found the condition as previously stated. An injection of $6 \mathrm{mg}$. of phenolsulphonephthalein showed the dye to appear on the right side in six minutes; left in nine minutes. The total amount recovered in two hours was: right 25 per cent., left 10 per cent. On account of the persistent pain an exploratory examination of the kidney was undertaken. Temperature at the time of operation was 102.2 , pulse 100 , leukocytosis 8,100 . The operation was performed Feb. 16, 1912. An anterior incision was made. The peritoneal cavity was opened and explored. Nothing abnormal was found. The peritoneum was closed and stripped toward the median line, exposing the left kidney retroperitoneally. The kidney was but little larger than normal, and several grayish depressed areas were seen beneath the capsules. On section there were many pale grayish, sclerotic areas, some near the cortex, others in the pyramid. The remainder of the kidney was reddish yellow, and there were acute red streaks near the apices of the pyramids.

In view of the history these sclerotic areas were considered to be old resolved foci of acute inflammation. It was believed that the persistent pain following the acute attack was probably to be explained by these areas, and the kidney was therefore removed. The fat about the pedicle was adherent to the vessels, and as they could not be cleaned the pedicle was clamped and the wound closed about the clamp, which was removed on the fourth day. The patient made an uneventful recovery except for an infection which took place in the wound.

Pathologic report is as follows: The surface presents several opaque, grayish areas which are depressed and contracted. Some of these areas are surrounded by a small area of injection, irregular in outline. On section several similar but smailer opaque areas not surrounded by areas of injection are seen scattered throughout the kidney substance. Near the upper pole is seen an area $2 \mathrm{~cm}$. in diameter, symmetrical and definite in outline, containing another area which is apparently a blood-clot. The capsule of the kidney strips easily, leaving a red surface in places. Cortex measures $6 \mathrm{~mm}$., and glomeruli are distinct. Histologic diagnosis is acute, diffuse inflammation of the kidney, with healed areas.

Three months after operation the patient was in the best health which she had enjoyed for many years. She had gained 34 pounds in weight. Eight months after operation excellent health continues. Two years and four months after operation the patient is in excellent condition.

C.sse 9.-Woman, aged 48, seen in consultation with Dr. H. Baker, March 2, 1912, presents a history of having had repeated attacks of pain in the left kidney region, associated with fever, chills and vomiting. These attacks confined her to bed for periods of weeks many times during the past five years. Since the first attack there has been constant pain in the left kidney region and during the past year the patient has been unable to lie down in bed and has been obliged to sleep sitting up on pillows. March 1, 1912, the patient was cystoscoped by Dr. Baker. The bladder was found to be rormal. Both kidneys were cauterized. The urine obtained from the right side was acutely red and showed fresh blood. with a few squamous and large round cells. The fresh blood was believed to have been caused by the catheter. The urine of the left side was slightly cloudy and showed a few pus cells, single and in clumps, many squamous, polygonal and large round cells, many clumped with fat drops adherent.

The patient was seen by me, March 4, 1912. She was a poorly nourished woman, weighing 110 pounds, and complained of severe pain in the left kidney region. There was tenderness in the left costovertebral angle. The temperature was 99.4 and the pulse 108 . As a result of the consultation it was decided that an exploratory operation of the kidney should be made.

March 10, 1912, the kidney was approached through an anterior incision, the peritoneal cavity opened and explored. Nothing abnormal was found. The peritoneal wound was closed and stripped toward the median line, exposing the kidney. The perirenal fat was slightly adherent to the kidney. This was freed and the kidney delivered. The organ was of normal size and rather pale. It was split in Brödel's line. The cut surface showed the tissue everywhere to be paler and firmer than normal, and there were no focal changes in the kidney tissue and the structure of the kidney was 
normal, other than for its pale character. It was decided not to remove the organ. The capsule was stripped, the kidney fixed in position, and the wound entirely closed.

The patient made an uneventful recovery. The wound healed by first intention, and two months following operation there is but slight pain of the old character, which has continued to come only after unusual exertion. The urine still contains polygonal and large round cells with a small amount of fat. Six months after operation the old pain had entirely disappeared and the urine contained but a few squamous epithelial cells.

It is believed that the pathologic process present in this kidney was a connective tissue repair of a diffuse inflammation involving the whole kidney. The kidney was left in this patient as more or less of an experiment.

This patient has had much relief, yet there is pain of varying degree in the region of the affected kidney, most distressing when fatigued, or when the urine becomes much concentrated. The pain may be considered annoying rather than severe. The patient considers herself much benefited but not cured by the operation. The urine examined many times shows renal cells and occasional granular casts; a small but variable number of leukocytes and sometimes fat globules. Her present symptoms do not warrant nephrectomy.

CASE 10.-Woman, aged 55, was operated on twelve years ago for carcinoma of the breast. Eleven years ago she had an attack of lobar pneumonia. She was admitted to the Long Island Hospital Fels. 19, 1914, complaining of pain in the pelvis with bloody discharge from vagina. Physical examination revealed the following defects: A diastolic murmur was heard at apex; not transmitted. Vaginal examination suggested carcinoma of the cervix, and a histologic examination of the tissue proved it to be carcinoma.

April 30, 1914, I performed an abdominal hysterectomy from which the patient made an uneventful recovery.

May 20,1914, the patient's temperature rose to 102 , white count 14,000 . Urine showed the slightest possible trace of albumin, a few squamous large and small round cells and a few leukocytes. The patient continued to run a high temperature and pulse; became extremely sick and eight days after the onset when seen by me the urine had become cloudy with considerable pus. There was tenderness and spasm over the whole right side of the abdomen and the right kidney could be felt considerably enlarged and extremely tender in front and in the costovertebral angle. Temperature 102, pulse 120 , respiration 30 . The kidney was exposed through the loin; the perirenal fat was extremely edematous; the kidney was considerably enlarged and of a bluish tint. Organ incised; surface acutely reddened throughout, otherwise negative. A rubber drainage tube was placed into the kidney pelvis and the cut surface closed about it. The external wound was closed about the drainage tube. The $B$. coli was recovered from the kidney incision and from the urine. The temperature dropped to normal within thirty-six hours and the patient's condition now, two weeks after operation, is satisfactory in every way.

CASE 11.-Boy, aged 19, referred by Dr. George Muttart, had negative past history, except that he had symptoms of acute appendicitis for three days. Appendectomy was performed by me July 23, 1912. Free cloudy fluid was found in the abdomen and pelvis, considerable in amount. Abdomen and pelvis were drained. Patient made an uneventful recovery; wound healed on eighteenth day; on twentieth day following operation, the patient had a rise of temperature to 102 associated with a chill. The temperature continued to range between 101 and 103 for five days without other subjective signs except that there was indefinite pain in the right kidney region.

On the sixth day after onset of these symptoms there was marked tenderness in the right costovertebral angle and rigidity of the right rectus muscle. The urine for the first time became slightly cloudy and contained a trace of albumin and some blood and pus. A cystoscopic examination showed the bladder to be normal. The urine from the left kidney was normal, that from the right slightly cloudy, containing pus, blood and the colon bacillus. The patient was becoming progressively sicker, temperature 103, pulse 110 .

The right kidney was exposed through the loin. The perirenal fat was filled with a cloudy serum, the kidney much enlarged and congested and beneath the capstule were seen many irregular areas of acute congestion. The kidney was split and these areas were found to be scattered through the kidney substances. A drainage tube was passed through the kidney into its pelvis and the kidney closed about the tube by mattress sutures. The external wound was closed about the drainage tube. The patient's temperature dropped to normal on the third day and the abnormal elements gradually disappeared from the urine. The patient is well almost two years following the operation.

During the past ten years I have seen seven patients in whom all signs pointed to a diffuse acute unilateral inflammatory process of the kidney, and in whom the symptoms subsided without operation. In four of these cases the colon bacillus was isolated from the affected kidney by means of the ureter catheter. The only sign of the disease remaining in these cases has been an indefinite pain in the region of the affected organ. In but one of these patients is this constant and in the others it may be considered absent, or present only during periods of fatigue. The urine in all shows but little abnormal. This type is illustrated by the following case, which is typical of this group:

CASE 12.-Woman, aged 23, seen in consultation with Dr. A. L. Stavely and Dr. F. R. Hagner at Washington, D. C. Jan. 14, 1914, for many months had some indefinite feeling of discomfort in the right side, especially after playing golf, but no importance had been attached to it. January 8 she was seized with a severe pain on the right side of the abdomen, a chill and fever. The suspicion of appendicitis was entertained. When seen by Dr. Stavely her temperature was 102 and there was marked tenderness over the right kidney region associated with mucle spasm. Further examination showed a small amount of pus in the urine, a leukocytosis of 30,000 and the colon bacillus was isolated in culture from the urine. The patient became progressively sicker, the temperature ranging between 100 and 103. A cystoscopic examination by Dr. Hagner showed a distinct reddening of the whole bladder mucosa. The urine from the right ureter showed numerous epithelial cells and a moderate number of pus cells; the urine from the left kidney was normal. The kidney was injected with argyrol as it was thought that the patient might have a pyelitis. The temperature the following day (four days after onset) reached 104. The leukocytosis was 21,000 ; the polymorphontuclear leukocytes were 84.5 per cent. The patient's general condition was much worse and although the physical side had changed but little, the patient had become progressively more septic and the intoxication became alarming. When seen by me, six days following the onset, the patient's temperature had dropped to normal and the urine contained a considerable amount of pus for the first time. Physical examination was negative except for tenderness over the kidney region, in front and in the back, there being tenderness still existing in the costovertebral angle. The treatment, which had consisted of forced fluids and large doses of hexamethylenamin, were continued. The patient gradtally improved and had no remissions of symptoms. At the present time, five months following the illness, the patient is in her usual health, although there is some indefinite discomfort in the right kidney region during fatigue. The urine is negative.

\section{CONCLUSION}

In the first class, the single kidney with multiple miliary abscess formation from which malignant toxemia results, the organ must be sacrificed in order to save the patient's life. In the second case of this class, mentioned in this report, only the central quarter 
of the kidney was involved. While this infected area was completely removed by a resection of the middle third of the kidney, and the healthy upper and lower thirds united successfully and the outcome of the case was most favorable, this procedure must be considered hazardous and cannot be recommended. In the other four cases of this class, here recorded, nephrectomy was performed, and it is felt that in each instance it was the only means which could have saved the patient's life. All these patients are living to-day and are free from symptoms, considering themselves well.

While house surgeon at the Boston City Hospital, I observed two cases of this form of the disease: one in which Dr. Paul Thorndike did a nephrectomy followed by recovery, and one in which Dr. F. S. Watson did a nephrectomy with drainage followed by death from progressive toxemia.

The proper treatment in the second class, the type of diffuse acute, unilateral inflammation of the kidney, cannot be so clearly defined and must depend on the course of the disease in the individual case. As a broad rule, it is felt that palliation, forcing fluids and the employment of urinary antiseptics, fortifying the patient's strength by a nutritious diet and stimulating drugs, should be instituted at the outset, and operative interference should only be undertaken when the symptoms and physical signs give evidence of progression to the point of seriously lowering the general resistance by toxic absorption. The time to operate in this class of cases requires experience with the disease and judgment in regard to the patient's general condition. Some of these patients recover without operation, as noted by Brewer, Cobb and others, and in the seven cases mentioned in this report.

When an operation is undertaken in this class, nephrectomy is not the only procedure to be employed. Favorable results have been obtained by simple decapsulation, ${ }^{1}$ by puncture of the infected areas with drainage ${ }^{2}$ and by splitting the kidney, closing the lidney wound by suture and decapsulating the organ or decapsulation and drainage of the kidney pelvis as in cases of this class recorded in this report.

On the other hand, when the symptoms and signs are of a distressing nature and the affected kidney is found to be the seat of old as well as new inflammatory processes or when the acute process is extensive, as in some of the cases of this report, nephrectomy is the best procedure.

40 Gloncester Street.

\section{ABSTRACT OF DISCUSSION}

ON PAPERS OF DRS. MAC GOWAN AND CUNNINGHAM

Dr. Hugh CaBot, Boston: The distinction between the hematogenous and the lymphatic types of infection is of importance, and $I$ believe it cannot be made from symptoms. From the symptoms alone we are likely to regard as hematogenous cases which are, in fact, lymphatic. The distinction has been made clearer to me by Dr. Cunningham's paper, and it turns sharply on the condition of the urine. I believe that, in practically all the cases in which there is evidence of a previous infection in the urinary tract, the infection of the kidney is lymplatic in origin. Mallory's work has shown that many of these cases of acute kidney infection follow mild cases of chronic pyelitis which have been entirely overlooked. In going over my records of cases of acute kidney infections I have been surprised to find how many gave a history of pyelitis, even many years ago, and

1. Cotton: Anu. Surg., November, 1911.

2. Brewer: 'rr. Am. Surg. Assn,, 1908, xxvi. some of these acute kidney conditions have originated in a pyelitis of childhood and are lymphatic in origin. The symptoms in Dr. Cunningham's cases are entirely out of proportion to the condition of the urine, because the condition is practically a closed one. It has little or no access to the tubules, and the urine does not show the extent of the damage. On the other hand, in the lymphatic cases secondary to a process in the pelvis, the condition of the urine follows quite accurately the symptoms. The cases that show very little change in the urine with marked general symptoms are likely to prove hematogenous, while the cases that give few symptoms and marked changes in the urine are of the lymphatic type. I was pleased to hear Dr. Cunningham lay great stress, in the treatment of these cases, on the desirability of great caution in the practice of nephrectomy. Modern surgery has shown that amputation, whether of an extremity or of a kidney, is an admission of weakness. Many kidneys have been taken out for conditions which would have recovered with a damaged kidney, but one far better than none. That does not apply to tuberculosis, but I am dealing only with infections from other causes. We should retain these kidneys to the last moment, and resort to nephrectomy only when the patient will die without it. In a considerable number of cases I have operated with the belief that I shouid be obliged to do a nephrectomy, and instead, have done some minor procedure, generally nephrotomy and occasionally decapsulation, with good results, though always with a damaged kidney. I do not believe that the majority of those cases are hematogenous. It has long been impossible to explain the lesions. They occur, as a rule, not in the glomeruli, but at the base of the pyramids in one group, and close to the pelvis in the other.

Dr. B. C. Corbus, Chicago: The work of Debré and Paraf, to which Dr. MacGowan made reference in his paper, consists in reversing the Wassermann reaction. We all know the difficulty of making a diagnosis in renal tuberculosis, both by looking for the bacilli in the urine and by guineapig inoculation. An animal is sensitized to the tubercle bacillus by injections of tuberculin, so that this animal contains specific antibodies for the tubercle bacillus. This "tubercle amboceptor" is used as an index, and the patient's urine contains the antigen. In the Wassermann an antigen looks for an unknown antibody. In the work of Debré and Paraf an antibody looks for an unknown antigen. The technic is simple. I have been working at it for a year, but failed until I could get a good antibody. One cannot get it from a tuberculous patient because his serum often contains both the antigen and antibody. If an animal is sensitized by injecting it with bovine tubercle bacilli you can get this specific antibody, and it is no trick at all to hook this back to the antigen in the urine. I have tried it in a few cases, but they are limited on account of the lack of material, but 1 hope later to have the pleasure of presenting this technic more fully.

Dr. M. Krotoszyner, San Francisco: Regarding Dr. MacGowan's statement that the cystoscopic evidence in renal tuberculosis is invariably to be found at or around the ureteral opening of the affected side, I recall a case of a tuberculous kidney in a woman of 42 , in whom the cystoscopic findings were entirely negative. On account of this and the persistent absence of tubercle bacilli in the urine sediment, the diagnosis of tuberculosis, which was strongly suspected, was abandoned and the kidney was exposed witlout the application of the guinea-pig test, which was, in all probability, the only clew to a correct preoperative diagnosis. Two foci of tuberculous caseation were afterward found in the removed kidney.

Dr. J. H. Cunningham, Jr., Boston: With regard to Dr. MacGowan's remarks on tuberculosis and the clear summarization of them by Dr. Cabot, there is much of interest. The fact that we have never as yet seen a healed tuberculous process in a kidney that can be proved to be such is of primary importance. The lymphatic infection of the kidney, spoken of by Dr. Cabot, is interesting and I believe our attitude with regard to the manner in which the tuberculous process is disseminated 
in the kidney should be somewhat changed. What seems to be neglected, in considering infections within the kidney, is the fact that the kidney is an organ which may disseminate disease through itself by the channels already existing. We may have a single focus develop in a kidney as a hemic or ascending infection, and from that single focus other parts of the organ may be infected by the kidney channels. The cases which form the basis of my paper are examples of this sequence of events. I believe we have too often considered multiple lesions in a kidney to be simultaneous in this organ when, in reality, there was a primary renal focus from which other parts of the organ had become infected through the lymphatics, blood-vessels or the spaces in the connective tissue stroma.

Dr. Granville MacGowan, Los Angeles: In this connection I will report a recent most interesting case. A man of 50 , with pyuria, had had repeated attacks of left-side renal colic and had passed, at different times, a number of small stones after such attacks. Their frequency and severity had invalided him. A roentgenogram showed two large stones in the left lower pole. I catheterized both ureters, and obtained a macroscopically clear urine, which contained only a little albumin and a few red blood-cells. The phthalein test for functional capacity of the left kidney was better than that of its fellow; they were both normal. The patient had a large tumor in the left flank. I could satisfactorily explain this only by surmising the existence of two ureters on the left side leading to a double kidney, and that I had overlooked one. Following an atrocious attack I cut down on this kidney and found a perinephritis which was like a wasp's nest or honeycomb. The fatty capsule of the kidney was adherent to everything about it and so intimately to the muscles along the spine and the descending colon that they had to be dissected directly from it. The kidney itself was a sound organ lying in the midst of this undrainable surrounding tissue. There was no stone; the shadows had been cast by two cavities filled with granulation tissue, pus and débris which lay in the lower part of this mass and communicated with the ureter. The agent of infection was the Staphylococcus aureus. The kidney was removed inside its capsule, and the multiple abscess cavities were dissected off the healthy tissue cn masse. The side had been injured two years previously by a blow from the crank of the patient's automobile. After the operation he was nauseated for days, and there was much pus in the urine for four days. On the ninth day, after a chill with a rise of temperature to $105 \mathrm{~F}$., followed by anuria, on examining the right flank $I$ found a tumor which proved to be a displaced kidney, probably dislocated by the retching following the first operation, causing a kink in the ureter. The kidney was exposed, delivered and decapsulated after pylorectomy to relieve pressure. Underneath the capsule of that kidney were hundreds of small miliary abscesses. The kidney presented by Dr. Cunningham reminds me of it. There was no abscess large enough to cut into and drain. The urine, which at the time of the preliminary examination had been normal, had during that nine days become infected. After decapsulation the kidney was suspended and drained for three days through the pelvis. At that time the patient's urine was perfectly clear, it contained no tube casts and no microscopic evidence of pus. I do not know, however, what the outcome will be.

Compiling Hospital Morbidity Statistics.-Bolduan has suggested a plan for compiting hospital morbidity statistics in this country. The method is especially applicable to the hospitals of a large city, but might be used for the hospitals of an entire state and is capable of being made nation wide in scope. The essential feature of the plan is the filling out of "discharge certificates," analogous to ordinary death certificates, on the discharge of each patient from a hospital. These discharge certificates are then sent to a central filing bureau, preferably the health department, and there classified and analyzed.-John W. Trask, Supplement No. 12, Public Health Reports, April 3, 1914.

\section{ALTOGENOUS VACCINES IN TREATMENT OF SCIATICA}

\author{
FRED. C. ZAPFFE, M.D. \\ CHICAGO
}

A man, aged 32, commercial traveler, consulted me because of an attack of sciatica (left-sided) which had continued practically unabated in the severity of the pain for eight months. During this time the patient had been under the continuous care of a number of physicians, but without more than slight temporary relief. He was kept from his work during all of this time, and, incidentally, lost not only time, but 60 pounds in weight. He was treated medicinally; the spinal nerve roots were injected with alcohol; he was given gonococcus vaccine; placed in a plaster-of-Paris cast; Buck's extension was applied to the leg; the Paquelin cautery was applied to the skin from spine to heel over the entire course of the sciatic nerve.

From these procedures he received only temporary relief. When I first saw him he was hardly able to walk because of the pain, nor could he lie down with comfort. He was in constant misery.

The history shows that he had an attack of gonorrhea six weeks before the sciatica began, and that about ten days after his doctor told him he was cured of his gonorrhea, he felt the first pain in the nerve. The clinical picture thereafter was characteristic of sciatica. There was no question about the correctness of the diagnosis. There was no evidence of a gonococcus metastasis elsewhere in the body.

Examination failed to find any pathologic condition except pain on pressure over the left sacral foramina. The spine could be put through the full range of motion, even violent motion, bending and rotating, without causing pain. Hipjoint disease was eliminated. Examination of the prostate gave negative findings. The teeth and gums were examined carefully and thoroughly by the patient's dentist. In short, there was no physical evidence of the cause of the sciatica, and yet when I took the temperature reading it was $99.6 \mathrm{~F}$. at 8 p. m.

The examination was continued systematically. A Wassermann test was made, with negative results. The tuberculin test was also negative. The chemical examination was likewise negative. Roentgenograms were made of the spine from top to bottom, and of both hips and the pelvis. Very careful examination failed to disclose any eridence of anomaly or disease.

A clue as to the possible nature of the trouble was obtained from the temperature record. The curve was very erratic, the temperature ranging between 98 and $99.8 \mathrm{~F}$., without any relationship to the time of day. The two-hour record was kept for a week and on not one day. was the temperature normal throughout the day. The tuberculin test did not influence the curve in any way.

A bacteriologic examination of the urine was now made, and staphylococci and a diphtheroid bacillus were found. A culture of these organisms was taken and a vaccine was made. It was a mixed autogenous raccine.

The first injection (hypodermically) of vaccine consisted of 100,000 bacteria. It produced a typical reaction. The patient felt slight chilly sensations; the temperature rose to $100.2 \mathrm{~F}$., and the pain in the leg was somewhat increased in severity. The reaction subsided in twenty-four hours. Four days later the second injection was given. It consisted of 250,000 bacteria. Again only a slight reaction was obtained. The third injection of 500,000 bacteria, given four days after the second, produced a very slight reaction, but the fourth injection of $1,000,000$ caused a decided reaction, far more marked than the first. Three more injections of $1,000,000$ each were given, the reaction being slight after the first, -and absent after the last two injections.

During the vaccine treatment the patient was encouraged to be outdoors, except for the first twenty-four hours after each injection; to eat well; to take some carefully prescribed exercise, and to observe personal hygiene thoroughly. $\mathrm{He}$ was given no medicine except magnesium citrate. The pain 